\title{
Udo Reifner Gewerkschaftliche Orientierung von Anwälten in Frankreich
}

Die große Streikbewegung in Frankreich im Mai I968 mit Fabrikbesetzungen, Besetzungen der Universitäten und Massendemonstrationen wurde auch für die Entwicklung der juristischen Profession in Frankreich ein wichtiges historisches Ereignis. Während vor 1968 das Recht und seine Hüter von den Gewerkschaften und von progressiven Theoretikern weitgehend als Domäne der herrschenden Klasse betrachtet wurde, die man besser insgesamt politisch umging oder auf deren revolutionäre Abschaffung man hoffte, ergab sich nach 1968 erstmals auch für die französischen Werktätigen in der Rechtsentwicklung eine progressive Perspektive. Den Arbeitern und Angestellten wurden durch eine sozialpolitische Gesetzgebung wichtige soziale Rechte eingeräumt, die die schrittweise paritätische Beteiligung von Gewerkschaftsvertretern an der erstinstanzlichen Arbeitsgerichtsbarkeit einschlossen. Außerdem setzte innerhalb des Juristenstandes selber ein umfassender Diskussionsprozeß ein, der zur Bildung von politisch bewußten und engagierten gewerkschaftlichen Organisationen von Juristen führte, die sich von den herkömmlichen Standesorganisationen absetzten.

I.

So bildete sich schon am 8 . Juni 1968 das »Syndicat De La Magistrature (SM), das es sich zur Aufgabe machte, über »die Unabhängigkeit der Richter zu wachen, für die notwendigen Reformen der Justiz und des Justizzugangs zu kämpfen und die beruflichen und gewerkschaftlichen Interessen seiner Mitglieder zu verteidigen «. (Artikel $3^{\circ}$ der "Statuts « vom 8. Juni 1968 i. d. F. vom 24. XI. 74). Diese Richtergewerkschaft, die inzwischen mehr als $25 \%$ der 4000 französischen Richter organisiert und die damit gleichberechtigt neben die Standesorganisation »Union Fédérale des Magistrats « getreten ist, hat in der Folgezeit auch in der Bundesrepublik dadurch von sich reden gemacht, daß sie sich hinter einen Untersuchungsrichter stellte, der das Untersuchungsgeheimnis gebrochen hatte, um die vielfältigen Abhängigkeiten in seiner richterlichen Arbeit zu denunzieren (Affäre Dujardin), sowie die Maßnahme eines Untersuchungsrichters deckte, der einen Arbeitgeber, bei dem innerhalb eines Jahres zwei Arbeiter wegen mangelnder Beachtung der Sicherheitsvorschriften

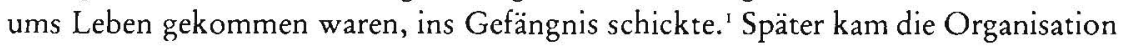

\footnotetext{
I Den schwierıgen Weg des Syndicat de la Magistrature zur Anerkennung als Gewerkschaft beschreibt Chavret, Sept ans de syndicalisme judicıaire, in: Pro Justıtı Nr. 9 "Les Juges», Brüssel o. J. S. I4 ff. Gerade an begrenzten Regelverletzungen wıe die Affären Dujardin (wo eın Journalist über die Arbeıt berıchten durfte), Obrego (der durch eine Umbesetzung von dem Posten des genchtlichen Revisors von Anwaltshonoraren entfernt wurde, weil er den Anwälten unbequem geworden war), Pascal (der die Benotungen seiner Amtshandlungen durch Vorgesetzte veröffentlichte) entdeckten die Rıchter, daß ihre
} 
"mouvement action judiciaire « hinzu, und 1973 bildete sich das "Syndicat des Avocats de France «, die beide im wesentlichen Anwälte organisieren. Auf Grund dieser Tatsachen änderten auch die Gewerkschaften, die in ihrer Mehrheit der rechtlichen Durchsetzung der Interessen der Lohnabhängigen skeptisch bis ablehnend gegenübergestanden hatten, ihre Politik. Die bei weitem größte französische Gewerkschaft, die CGT (über 2 Millionen Mitglieder) ${ }^{\text {ra }}$, stellte auf ihrer "Conférence Nationale Juridique " vom 28, 2. 1969 fest, daß "mit den neu erworbenen Rechten durch die großen Kämpfe vom Mai und Juni das französische Arbeitsrecht eine Reihe von Garantien enthält [...], die es an die erste Stelle der kapitalistischen Länder hebt «. Hieraus leitete der Kongreß »eine neue Verantwortung der Gewerkschaften gegenüber der Arbeiterklasse « ab, die darauf abzielt, die "juristischen Aktivitäten sowie das Interesse an juristischen Fragen in einer modernen Gewerkschaft zu perfektionieren «, um »das Erreichte gegen alle Versuche seiner Zurücknahme zu verteidigen «. ${ }^{2}$ Dabei geht die CGT davon aus, daß in einem kapitalistischen Staat niemals Rechte der Arbeiterklasse endgültig erworben würden, und daß die rechtliche Verteidigung integraler Bestandteil des gewerkschaftlichen Kampfes sei und in ihrer Bedeutung wie in ihrer Organisation »das letzte Glied der Kette von Maßnahmen zu sein hat, die gegen den Unternehmer gerichtet werden «. ${ }^{3}$ Die CFDT, die zweitgrößte Gewerkschaft Frankreichs, engagierte sich bei der Abwehr justizförmig vermittelter politischer Disziplinierungen. So wurden auf regionaler Ebene eine Vielzahl von Kontakten geknüpft, die z. B. zu gemeinsamen Kampagnen mit dem »Mouvement Action Judiciaire (MAJ) führten, die wiederum Hilfestellungen bei Werksbesetzungen und anderen vor allem strafrechtlich relevanten Formen des Arbeitskampfes gaben. In der Affäre Dujardin verteidigte die "Union régionale CFDT de Lille a als erste gewerkschaftliche Freiheiten auch der Richter. Das hat in der Folge zu partieller Kooperation mit dem »Syndikat de la Magistrature " geführt. Gleichwohl dürfte bei der CFDT noch die größte Skepsis gegenüber einer Verrechtlichung der Interessendurchsetzung bestehen.

Ihre theoretische Widerspiegelung fand die rechtspolitische Neuorientierung in verschiedenen Publikationen. Das Organ des "Syndicat de la Magistrature «, "Justice«, versucht kontinuierlich eine vor allem politisch-moralisch getragene Justizkritik an praktischen Beispielen zu entwickeln und zum Teil in satirischer Selbstironie den politischen Standort der französischen Richterschaft zu analysieren. In ihrem Buch »Au Nom Du Peuple Français ... « ${ }^{4}$ hat ein Kollektiv von Richtern im Namen der SM »das Ghetto verlassen «s und einen umfassenden Bericht zur Lage und Funktion der Richterschaft und Justiz vorgelegt. Texier vom Vorstand des SM schreibt in seiner Stellungsnahme ${ }^{6}$ zur Kritik an dieser Publikation: »Das Recht ist für uns eines unter vielen Instrumenten in einer Gesellschaft der Konflikte, in der jede Partei versucht, dieses Instrument am besten für sich zu nutzen . . . Wir müssen dabei feststellen und bedauern, daß es dabei nicht immer auf der Seite der Schwächsten und der Schutzbedürftigen ist. . . . Die Arbeiter haben immer die Justiz als im Dienste anderer als ihrer Interessen stehend angesehen. Heute diskutieren sie mit uns, um uns das besser verstehen zu machen, was unsere Professoren uns an der

Standesorganısatıonen nıcht willens und in der Lage waren, ihre materıelle Unabhängıgkeıt zu verteıdigen.

ı Der Leıter der jurıstıschen Abteilung, Jean Schaeffer, ıst Mitglied der Sozıalistıschen Parteı.

2 Nach Le Metallo, Dezember 1969, S. 1/2.

3 Ebd., S. 3 .

4 Syndicat de la Magistrature, Parıs 1974, Lutcer/Stock 2.

5 Ebd., S. 7.

6 Gazette du Palais du I2./13. Februar 1975, S. 22. 
Fakultät verborgen halten konnten«. Diese Hinwendung zum Konkreten wird in derselben Stellungnahme unterstrichen: „Unsere Selbstreflektion lief Gefahr, sich in Narzismus zu entwickeln. Deshalb haben wir als Kontrapunkt hierzu auf jedem unserer Kongresse das Thema >Gesetz aufgeworfen, nicht in den abstrakten Kategorien der Universität, sondern an Hand der konkreten Fälle, die unsere Mitglieder erlebt und berichtet haben «.

Demgegenüber erfolgte in der Zeitschrift »actes « des »Mouvement d'Action Judiciaire « eine abstrakt theoretische Diskussion über das Recht, bei der die »kritische Analyse der herrschenden Rechtsideologie ${ }^{6 a}{ }^{6}$ als eine Demaskierung der Justiz, die "hierarchisch, kulturell isoliert, sozial abgeschlossen mit ihren Verkleidungen, Riten und ihrer Sparche unwandelbar ist ${ }^{7}$, im Vordergrund stand. Theoretische Anleihen werden dabei vor allem bei Poulantzas ${ }^{8}$ und Althusser ${ }^{9}$ gemacht. Eine umfassende theoretische Antwort auf diese Positionen haben M. und R. Wey] zu geben versucht $^{10}$. Sie lehnen das Recht als idealtypischen Ausdruck kapitalistischer Ideologie ab und begreifen die Rechtsentwicklung selber entsprechend dem Kräfteverhältnis als die dialektische Entfaltung des sozialistischen Rechts im bürgerlichen Recht, wobei sie hierdurch versuchen, als reformistisch und rechtsillusionär gegeißelte Forderungen zur Reform des Rechtes und der Justiz als notwendigen Teil des Ubergangs zum Sozialismus auszuweisen ${ }^{\prime \prime}$. Diese theoretische Auseinandersetzung, die durchaus ihren praktischen Ausdruck in den verschiedenen Organisationsformen und -zielen sich links verstehender Juristen findet, ähnelt dabei den entsprechenden Kontroversen innerhalb der theoretischen Diskussion um die Position eines progressiven Juristen ${ }^{\mathrm{r} 2}$ bei uns.

Ebensowenig aber, wie die im Grunde doch wenigen neuen Rechte der Arbeiter nach 1968 die eigentliche Ursache einer juristischen Besinnung der Gewerkschaften waren, so wenig können auch diese theoretischen Diskussionen als Grundlage der umfassenden Bewegung des Zusammenschlusses fortschrittlicher Juristen in Frankreich verstanden werden. Die tieferen Ursachen liegen vielmehr darin, daß auch in Frankreich, ebenso wie in ganz Westeuropa die Justiz in eine umfassende Krise, sowohl was die Effizienz ihrer Organisation, als auch was die Legitimation ihrer Veränderung gemessen an ihren eigenen Postulaten anbetrifft, geraten ist. Die tatsächliche Veränderung der Berufspraxis der Juristen selber war dabei die zunächst ebenso wie in der Bundesrepublik wesentliche Erscheinung, die nur in Frankreich im Kontext einer klassenbewußten politisch wie gewerkschaftlich organisierten Arbeiterschaft mit ungleich größerer Macht und Geschwindigkeit als in der Bundesrepublik den Betroffenen zum Bewußtsein gekommen ist. Einige dieser konkreten Entwicklungen sollen an Hand der Veränderung anwaltlicher Berufspraxis in Frankreich insoweit skizziert werden, wie es zum Verständnis der beiden

6a So die ergene Zielsetzung in Actes 4/74, S. 42.

7 Leclerc, La Justice et l'Avocat, actes $4 / 1974$, S. 9

8 Pouvorr politıque et classes sociales, Parıs 1972; dt.: Politısche Macht und gesellschaftliche Klassen, 2. Aufl. 1975.

9 Idéologıe et appareils ıdéologıques d'État; dt.: Ideologıe und ıdeologısche Staatsapparate, in Althusser, Marxısmus und Ideologie, Westberlin 1973, S. 11 i ff.

10 La Part du Drott dans la Réalité et dans l'Actıon, Paris $1968 \mathrm{~m}$. Nchw. der Aufsätze dieser Autoren; sowıe Révolution et Perspectives du Droit, Parıs 1974.

I I Insb. Revolution.. ebd. S. $149 \mathrm{ff}$., wo allerdings m. E. kaum überzeugend ın einer Kontraktualisıerung der Rechtsbezıehungen das sozıalistısche Recht "der Garantse" gesehen wird, das das kapitalisusche Klassenrecht abgelöst hat.

12 Vgl. die Kontroverse Ridder/Preuß zur Funktıon eınes Zusammenschlusses gesellschaftlicher Jurısten und von Juristen überhaupt, in KJ 71,$378 ; 72,16$; sow1e Verfassungsreformen und gesellschaftliche Aufgabe der Juristen, 71, 371, womit die Diskussion begann; vgl. ferner die Kontroverse Geulen/Stuby in Stuby, Diszıplinıerung der Wissenschaft, 1970 
nachfolgend beschriebenen Formen kollektiver und individueller Reaktion auf diese Entwicklung notwendig ist.

II.

Die herkömmliche französische Anwaltspraxis ist gekennzeichnet durch die Persönlichkeit des Anwalts, um dessen Person herum in der liberalen Tradition des unabhängigen Parteivertreters sich die Mittel seiner Berufsausübung gruppieren. Der übergroße Schreibtisch im Arbeitszimmer verstärkt jenen Glanz um die Person des alleinarbeitenden Anwalts, der sich im Gebrauch der Sprache im Schriftverkehr ${ }^{13}$ und unter Kollegen (notorische Briefanrede: Mon cher Maître) fortsetzt und im (obligatorischen) Plädoyer mit seinen umständlichen Referenzen an die Gegenpartei (mon bien estimé confrère) vor Gericht gipfelt. Die zeitraubenden Verfahren vor den Gerichten, das beim Cour d'appel (OLG) nicht vom avocat, sondern noch von dem zustäzlich bestellten avoué $\mathrm{e}^{14} \mathrm{zu}$ vollziehen ist, sowie der formalisierte Aufbau der Klageschrift und Klageerwiderung (jeder Satz hat mit attendue que zu beginnen und der Tenor wird am Ende mit der Formel "par ces Motifs... « eingeleitet), ist das adäquate Pendant zur magieverwandten Umständlichkeit anwaltlicher Berufsausübung und Ineffizienz.

Demgegenüber steht eine einschneidende Verschlechterung der sozialen Bedingungen der Anwälte. Die großen Firmen unterhaiten ihre eigenen Rechtsabteilungen und Treuhandgesellschaften, die es ihnen erlauben, untereinander weitgehend die kostspieligen Prozesse vor den staatlichen Gerichten zu sparen und Konflikte auf anderen Wegen zu regeln, was für einige wenige Anwälte bedeutet, »die Robe mit dem Attaché-Koffer und das Plädoyer mit dem >briefing « ${ }^{15}$ zu vertauschen. Die wenigen Prozesse innerhalb der Geschäftswelt, die noch zu führen sind, werden bei einigen Anwaltssozietäten konzentriert, deren Arbeitsstil und Einkommen von den Auftraggebern kontrolliert wird. Die Masse der Prozesse aber konzentriert sich immer mehr bei den unteren Einkommensschichten. Trotz Freiheit in der Honorarvereinbarung zwingt die materielle Lage der Klienten sowie die wachsende Zahl der Anwälte (ca. 10000 , davon ca. 7000 in Paris, zusätzlich 840 Avoués, 6330 Notare und 3500 Rechtsberater) dazu, sich mit Beträgen zufriedenzugeben, die nach Abzug der vom Anwalt zu zahlenden Verfahrensgebühren, die steigenden Fixkosten eines Anwaltsbüros nicht mehr auffangen können. Diese ergeben sich insbesondere aus den steigenden Mieten der anwaltlichen Büros in der City, steigenden Personalkosten und vor allem der Notwendigkeit kostspieliger Ausstattung und Unterhaltung von Büromaschinen und Einrichtungen, angefangen von der elektrischen Schreibmaschine bis hin zu Kleincomputern, die die zunehmende Masse von Kleinprozessen, mit denen der abnehmende Einzelerlös kompensiert werden muß, bewältigen helfen sollen ${ }^{16}$. Hinzukommt, daß die Vielzahl der Kleinprozesse die ständige Präsenz des Anwalts am Gericht erfordert, was bei den Pariser Verkehrsverhältnissen praktisch eine Büroarbeit zwischen 9.00 und 17.00 Uhr ausschließt, da die Termine nicht mit der Arbeitszeit des Anwalts abgestimmt werden können. Bei den Arbeitsgerichten wird wegen der hohen Arbeitsbelastung bereits bis $2.00 \mathrm{Uhr}$ morgens verhandelt, ohne daß der Anwalt wissen kann, wann er an der Reihe ist. Da

13 Für eıne eınfache Mitceilung wırd eıne Seıte benötıgt.

14 Ansonsten ist diese Zwerteilung neuerdings abgeschafft.

is Leclerc, La Justice et l'A vocat, actes $4 / 1974$, S. 9 (11).

I6 Dazu Syndicat de la magıstrature, Au nom du peuple françats, 1974, S. I $22 \mathrm{f}$. 
die Großzahl der Anwälte zudem auch auf die Solvenz der ärmeren Schichten angewiesen ist, gerade wenn diese in ökonomischen $\mathrm{Krisen}^{17}$ stecken, bildet das vollständig unzureichende Armenrecht ${ }^{18}$ eine weitere starre Grenze bei steigenden Kosten. Unter diesen Bedingungen wird auch die Rente der Anwälte, die unterschiedslos nach 40jähriger Berufsausübung I 210 frs (ca. 650,- DM) monatlich beträgt, zum einzigen, aber offensichtlich vollständig unzureichenden Mittel der Alterssicherung ${ }^{19}$.

Dieser ökonomischen Krise des alleinstehenden französischen Rechtsanwaltes begegnen die Geschäftswelt, gewisse Geschäftsanwälte sowie der französische Staat mit den Mittel der Arbeits»effektivierung «. Über Treuhandgesellschaften werden bestimmte Anwaltsbüros direkt an die Wirtschaft angeschlossen ${ }^{20}$, die sie (allerdings noch unter dem Nichtigkeitsverdikt der Gerichte ${ }^{21}$ in Kapitalgesellschaften zu transformieren sucht. Geschäftsanwälte stellen unter dem formalen Mantel der Sozietät junge Anwälte für I $500 \mathrm{frs}(=800,-\mathrm{DM})$ im Monat ein. Diese sind de facto lohnabhängig ohne eigenes Telefon und das Recht der eigenen Klientel, wobei sie davon »träumen, die Stufenleiter zur Chefétage eines Tages erklommen zu haben * (Leclerc a.a.O., S. I I). Hierdurch wird die effektive Ausnutzung des Büros zugunsten der wenigen Stammanwälte gewährleistet ${ }^{22}$. Der Staat schließlich leistet durch eine großangelegte Uniformierung der Justiztätigkeit (Fusion der Anwaltsberufe: Avocat, Avoué, conseil juridique, fiduciaire) und Prozeßvereinfachung (Reduktion der Mündlichkeit des Verfahrens; Vordringen der Inquisitionsmaxime; Vordringen der Rolle der Sachverständigen, die ohnehin als kompetent für jegliche Beweiserhebung den französischen Zivilprozeß beherrschen) die gesetzliche Hilfestellung zu diesen Prozessen. Diese »kapitalistische« Lösung der ökonomischen Krise des französischen Anwaltsstandes führt dabei gleichzeitig zu einer veränderten Einstellung zur Klientel. Die wenigen Großunternehmen und Wohnungsbaugesellschaften, die in ihrer Geschäftspolitik eine steigende Zahl von Prozessen mit Arbeitnehmern und Mietern zu kalkulieren haben, sind in der Lage, solche Anwaltssozietäten vollständig zu beschäftigen und damit de facto von der Situation des Kunden in die Situation des Arbeitgebers zu gelangen. Die Anwälte, die nunmehr ständig für ein Unternehmen nicht nur die juristische Transformation vorgegebener Interessen zu vollziehen haben, sondern notwendig in den gesamten ökonomisch-politischen Prozeß der Interessenvertretung für ihre Auftraggeber eingespannt werden, führen zu einer Politisierung anwaltlicher Tätigkeit von seiten der Unternehmen.

Vor allem im Arbeits- und Mietrecht, in dem den wenigen Großunternehmen stärker werdende Zusammenschlüsse (CGT und CFDT; CNL) der Arbeiter und Mieter die Alleinherrschaft streitig machen, läßt sich der Einzelprozeß nicht mehr ohne Rücksicht auf die allgemeine Entwicklung des politisch-sozialen Kräfteverhältnisses führen. Diese Entwicklung zeigt sich auch in der Politisierung der Rechtsprechung selber an, die sich durch die Generalklauseln ${ }^{23}$ gezwungen sieht, die politischen Argumente und die politische Glaubwürdigkeit der Anwälte in ihre Entscheidungen einzubeziehen. $\mathrm{Da}$ im Arbeitsrecht die erste Instanz weitgehend aus paritätisch besetzten Laienkammern besteht, tritt die rein juristische Argumen-

17 Kündigung des Arbeits- oder Mietverhältnısses etc.

I 8 Aide judiciaıre, Berıcht des SAF von seınem Kongreß in Grenoble 9./ro./1r. Nov. 1974.

I9 A. a. O. "Résolution sur les Retraites".

$20 \mathrm{Vgl}$. a. a. O. Motıon sur la Patrımonalité et la Sécurité, sowıe Kessous, La Défense vue par un Magıstrat, actes $4 / 74$, S. $18(20)$.

2r Vgl. Gazette du Palais I 974 v. 6. Sept., S. I8.

22 In den USA soll es bereıts Sozıetäten dieser Art mit über roo Anwälten geben.

23 Bes arbeitsrechtlicher Kündigung etwa die causes réelles et sérıeuses oder der raıson économıque. 
tation in den Hintergrund. Anwaltssozietäten, die vollständig in die sie beschäftigenden Unternehmen einbezogen sind, haben daher eine ganz andere Effektivität in der Vertretung ihres Klienten als der Anwalt, der sich die notwendigen Informationen von den einzelnen, juristisch ungebildeten Arbeitnehmern zusammensuchen muß, der sich ohne Kenntnis des Kräfteverhältnisses in bestimmten gesellschaftlichen Bereichen auf die konkrete Situation zu beschränken hat und daher ohne Kontakt zu den Gewerkschaften und Mieterverbänden dem Richter als gefahrloser Verlierer erscheinen muß.

Auch im Strafprozeß erkennen die Anwälte immer mehr, wie im Grunde peripher ihre Arbeit bleiben muß, wenn sie als Verteidiger den vielen kleinen Dieben als Führer durch den Dschungel des justizmäßigen Verfahrens dienen müssen, ohne ihnen wirklich mangels zeitlicher und finanzieller Möglichkeiten helfen zu können, während die Fälle, in denen einmal die Kriminalität von Unternehmern, Arbeitgebern und Finanz»genies « die Gerichte beschäftigen, schon im Vorfeld z. T. politisch bereinigt werden. So schreibt ein französischer Richter über die Strafverteidigung: "Die Verteidigung ist heute Sache eines Spezialisten, dessen bevorzugtes Arbeitsgebiet das justizmäßige Verfahren ist mit seinem Großen Zirkus, dem Gericht... . Aber in dem Augenblick, in dem die den Ablauf des Strafverfahrens letztlich bestimmenden Kräfte ansetzen, sind der Anwalt und das Recht abwesend. Das Recht kommt erst später hinzu, um die Position des Stärkeren zu unterstützen ${ }^{24}$. Wer sich hiergegen auflehnt und nicht dem Gesetz des schlechten Verdienstes beim »kleinen Mann" das Gesetz der Masse der behandelten Fälle entgegensetzt, sondern sich, sei es aus humanitären, religiösen oder politischen Gründen, im Einzelfall entsprechend seiner sozialen und nicht seiner finanziellen Relevanz engagiert, muß auch in der Strafverteidigung bald aufgeben, um seine Anstellung in einer "Anwaltsfabrik « zu suchen, in der Verteidigung, nach Umfang und Qualität durch die Höhe des Entgelts genormt, am Fließband produziert wird. Mit der Integration der Anwaltspraxis in die Strukturen auf Gewinn ausgerichteter Unternehmen wird somit in der Tat für einige Geschäftsanwälte die Möglichkeit geboten, sich politisch konkordant für die Unternehmen bei hoher finanzieller Rentabilität einsetzen zu können.

Diese Sanierung geht jedoch in gleicher Weise auf Kosten der Masse der Anwälte, die ihre Arbeitskraft unter dem Qualifikationsniveau zu verkaufen haben und denen das Recht selbstbestimmter und eigenverantwortlicher Berufspraxis genommen wird, wie auch auf Kosten der Mehrheit der Bevölkerung, deren Interessen durch die anwaltliche Maschinerie eines Großbüros nur noch formalisiert vertreten werden. Die Konzentration weniger Großpraxen im Stadtzentrum und in Gerichtsnähe erschwert noch zusätzlich den persönlichen Kontakt, der bisher die Basis der anwaltlichen Vertretungsmacht war. Das Problem anwaltlicher Unabhängigkeit hat durch diese ökonomische Integration einiger Sozietäten in die Geschäftswelt inzwischen seine ganze politische Dimension entfaltet. Selbst der Kongreß der anwaltlichen Standesorganisation »Associatión Nationale des Avocats « (ANA) fragte sich 1974: "Darf der Anwalt engagiert sein ${ }^{25}$ oder beraubt er sich damit der "Freiheit ..., die ihm ein ungeheures Aktionsfeld eröffnet? ${ }^{26}$. Diese Freiheit der sachlichen Indifferenz entpuppt sich immer stärker als eine Parteinahme für die Seite, für die das Gesetz und die juristische Form oder Argumentation und Rechtsverwirkli-

\footnotetext{
24 Kessous, La Défense vue par un Magistrat, actes $4 / 1974$, S. I9.

25 Farthouart auf dem Kongreß der (konservativen) Association Natıonale des Avocats I974, abgedruckt in actes Okt. 74, S. 16.

26 Batonnier Chaplet nach actes ebd.
} 
chung Partei ergriffen hat. Für die sozialen Belange der rechtlich schwächeren Schichten hat diese Unabhängigkeit die Verstärkung ihrer eigenen Ohnmacht zur Folge. ${ }^{27}$

Erlaubte die Berufsideologie, durch einfache Bezahlung ein unbewußtes Engagement des Anwalts zu erkaufen, so prüfen heute viele französische Anwälte, ob sie das Mandat auch im eigenen politischen und sozialen Interesse vertreten wollen und inwieweit das entsprechende Mandat eine ausreichende Bezahlung sichert. Ohne Komplize des Mandanten werden zu müssen, geben dabei die Vertretungen der großen Masse der Bevölkerung bei weitem größeren Raum, für grundsätzliche Rechte der Person, individuelle Freiheit und soziale Gerechtigkeit auch in der Robe eintreten zu können. Durch die Entlohnungsbedingungen wird der Einzelanwalt jedoch in wachsendem Maße dann in Widerspruch zu seinen eigenen finanziellen Interessen gebracht, wenn er durch die Veränderung des Arbeitsinhalts von einer "defensiven (Schutz der Rechte) zu einer verantwortlichen, mutigen und offensiven Konzeption ${ }^{28}$ im $»$ Interesse der großen Mehrheit der Franzosen ${ }^{29}$ kommen will.

III.

Die erste Reaktion der Anwaltschaft auf die ökonomische Entwicklung war und ist zum großen Teil konservativ: Wahrung des liberalen Besitzstandes, Verbot der Großpraxis mit mehr als s Anwälten, Verbot des versteckten Klientelverkaufs etc., nachzulesen im »règlement intérieure des Barreaux de Dijon «. Der Cour d'Appel de Dijon hat die Klausel mit der Beschränkung auf $s$ Anwälte für nichtig erklärt ${ }^{30}$.

Der Weg zurück zur Einzelpraxis ist jedoch nicht nur rechtlich versperrt, sondern vom Gesichtspunkt der Arbeitseffizienz her nicht gangbar, soll der Anwaltstand nicht allein durch ein System staatlicher Subventionen direkter oder indirekter Art aufrechterhalten werden. Um dieser Krise des Anwaltsstandes zu begegnen, haben die französischen Anwälte sowohl auf kollektiver als auch auf individueller Ebene nach Antworten gesucht.

a) Die Vielzahl der Anwaltsorganisationen, die in Frankreich bestehen, zeigen nicht nur den Willen und Zwang, berufliche Interessen kollektiv zu vertreten, sondern sind in ihrer Vielzahl Ausdruck der unterschiedlichen sozialen Situation und politisch-ökonomischen Interessenrichtungen.

Während die "Association Nationale des Avocats« und die "Réunion der Nouveaux Avocats de France " (RNA) ${ }^{3 r}$ mehr oder weniger Standesorganisationen sind, die in ihrer eigenen Struktur die allgemeine Einkommensverteilung zwischen wenigen Spitzenanwälten und der Masse der Anwälte spiegeln und entsprechend nur die ökonomischen Interessen des abstrakten Anwalts an sich vertreten, ist in der "Fédération Nationale des Unions des Jeunes Avocats« (FNUJA) ein recht loser Zusammenschluß vieler lokaler Interessenvertretungen der vor allem benachteiligten jungen Anwälte erfolgt. Ihre besondere Situation ergibt sich daraus, daß sie als Anwaltsreferendare (avocats stagiaires) rechtlich schon vollwertige Anwälte sind, gleichzeitig aber lohnabhängig und in prüfungsbedingter Abhängigkeit zum ausbildenden Anwalt und der Anwaltskammer bleiben. Die FNUJA ähnelt dabei einem

27 Vgl. Farthouart ebd. S. I5.

28 Jacob, Präsıdent des Syndicat des Avocats de France (SAF), Rede auf dem Kongreß 1974 in Grenoble (Anm. 18).

29 Ebd.

30 Gazette du Palais v. ir. September 1974, S. 17

3r Organisation der inzwischen in den Anwaltsstand überführten "avoués". 
Zusammenschluß von Vereinen, in denen Interessenvertretung, Kommunikation und spontane Einzelinitiativen einen bestimmten Rahmen gefunden haben.

Im "Mouvement Action Judiciaire (MAJ) hat sich dagegen eine zahlenmäßig wesentlich unbedeutendere politische Form des Zusammenschlusses von Anwälten und Jurastudenten gebildet, die "sich zum Ziel gesetzt hat, politisch an der Seite der Arbeiter die juristische Phase der sozialen und politischen Kämpfe unter Ausnutzung der positiven Aspekte des Rechts und der internen Widersprüche der Justiz anzufachen ${ }^{32}$. Ihre Aktionen liegen im wesentlichen in einer allgemeinpolitischen, z. T. spontanen und spektakulären Arbeit, bei der politische Prozesse, rechtlicher Beistand bei Werksbesetzungen und Streiks sowie politische Agitation zu einer von der Normalität abweichenden ausgewählt politischen Berufspraxis führen soll ${ }^{33}$.

Am I 5 . Mai 1973 hat sich erstmals mit dem "Syndikat des Avocats de France (SAF) ein Zusammenschluß von Anwälten gebildet, der zum Ziel hat, in "gewerkschaftlicher Orientierung die ökonomischen Bedingungen der Existenz und vollen Berufsausübung der Anwälte ${ }^{34} \mathrm{zu}$ sichern.

Diese ökonomische Interessenvertretung stellt sich dabei in direkten Gegensatz zur herkömmlichen Standespolitik der Anwaltsvereine, indem sie deutlich werden läßt, daß die "Verteidigung der ökonomischen Subsistenzmittel des Anwaltsberufes als wesentliche Kampfaufgabe für das Überleben des Anwalts «3s auch gegen eine Minderheit von Geschäfts-Anwälten gerichtet sein muß, die "mit Genugtuung die Fusion im Anwaltsstand und ihrem Anschluß an den Finanzsektor ${ }^{36}$ sehen würden. Nicht mehr der abstrakte Anwalt an sich, sondern die konkreten Interessen der Mehrzahl der Anwälte sind Ausgangspunkt dieser Organisation. Die ökonomische Interessenvertretung der Anwälte soll nicht als verantwortungsloser Standesegoismus betrieben werden, sondern unter der Zielsetzung eines allgemeinen gesellschaftlichen Fortschritts in der anwaltlichen Dienstleistung. Mit dem anwaltlichen Engagement soll nach den Worten des Präsidenten Jacob »der Beweis angetreten werden, daß die Interessen der großen Mehrheit der Franzosen mit den Interessen dieser Anwälte übereinstimmen « ${ }^{37}$. Nach den Statuten des SAF steht die ökonomische Zielsetzung daher im Zusammenhang mit vier weiteren Zielen:

I. Verteidigung der Unabhängigkeit der Anwaltskammern,

2. Ausweiten der Rechte und Möglichkeiten anwaltlicher Verteidigung,

3. Okonomische Existenzsicherung (Renten und soziale Sicherung) sowie Sicherung des Zugangs zum Anwaltsberuf,

4. Gemeinsame Aktionen mit anderen Berufsorganisationen des Justizbereiches für eine bessere Justiz,

5. Teilnahme an Initiativen für eine demokratische und bürgernähere Justiz sowie für die Garantie der öffentlichen und individuellen Freiheit.

Das SAF hat sich daher in der kurzen Zeit seines Bestehens nicht nur darauf beschränkt, etwa die jungen Anwälte und vor allem die avocats stagiaires für die Forderungen nach garantiertem Mindestlohn und Mindestarbeitsbedingungen zu organisieren, sondern hat zu Projekten der Gesetzgebung, zu Verletzungen von Bürgerrechten durch die Verwaltung sowie zu Veränderungen des Justizapparates

\footnotetext{
32 Actes $4 / 74$, S. 42 .

33 Eine Entsprechung zu ihr könnte man am ehesten in der »Roten Hilfe « In West-Berlin sehen, wenngleich die MAJ mit einer eigenen Zeıtung beı wettem eın größeres Gewicht in der Offentlichkeıt hat. Unmittelbare berufliche Interessenvertretung wird von dieser Organisation als reaktıonär abgelehnt. Vgl. im übrigen Vingtras, Rote Hilfe und politusche Justız in Frankreich, KJ 72, S. 24.

34 Gründungsaufruf "Pour une vérıtable organisatıon syndicale de défense de la professıon d'avocat $\alpha$. 35 Jacob, président du SAF Congrès de Grenoble 1974, S. 4.

36 A. a. O., S. 5.

37 A. a. O., S. 4
} 
eine breite Offentlichkeitsarbeit mit öffentlichen Diskussionen, gemeinsamen Veranstaltungen sowie Fernseh- und Presseerklärungen betrieben. So fand etwa aus Anlaß des Vorgehens der sogenannten "Anti-Gang «-Polizeibrigade gegen zwei algerische Anwälte, in der Rassismus und Mißachtung von Bürgerrechten im Verhalten der Polizei nachgewiesen wurde, eine Großveranstaltung mit 10 anderen Organisationen aller politischen Richtungen zum Thema: „Défense des Libertés et Pouvoirs de Police « statt..$^{73}$

Auf dem ersten nationalen Kongreß in Grenoble hat das SAF sein politisch-soziales Programm präzisiert. Zur Frage des Anwaltseinkommens hat es sich für eine Offenlegung der Honorarkriterien und eine einverständliche Tarifierung eingesetzt, jegliche staatliche Gebührenordnung, von der man nur eine autoritäre Fixierung der bestehenden Ungerechtigkeiten erwartet, jedoch abgelehnt. Gleichzeitig wurde eine Ausweitung des Armenrechts sowie die Verbesserung der Anwaltsrenten und ihre gerechte Verteilung gefordert. Der Kongreß wandte sich gegen die kapitalistische Integration des Anwaltsberufes, gegen Klientelzession und einseitige Abhängigkeit. Dabei blieb jedoch in der Frage der positiven Umorganisation der Berufspraxis eine gewisse Ambiguität, die sich im Nebeneinander von jungen Anwälten mit neuen vor allem kollektiven Arbeitsformen und älteren der liberalen Tradition verhafteten Anwälten zeigte.

Mit Stellungnahmen zum Arbeitsrecht, zum Pressewesen, zu den Problemen der Datenverarbeitung bei den Gerichten und richterlicher Unabhängigkeit hat der Kongreß die Arbeit der einzelnen Sektionen zu justizpolitischen Themen zusammengefaßt und die Offentlichkeit auf die Inhaftierung von zwei demokratischen Anwälten in Spanien hingewiesen. Damit wird auch die internationale Verantwortung des SAF deutlich, die durch eine Vielzahl von Kontakten vor allem zu italienischen und belgischen Organisationen unterstrichen ist.

Die SAF, die nicht verheimlicht, daß sie im "programme commun« der französischen Linksparteien eine Vielzahl ihrer Forderungen verwirklicht sieht, hat in den beiden Jahren eine erstaunliche Repräsentanz erreicht. Sie ist mit Sektionen in allen großen Städten vertreten, die durch 23 Anwälte im Rat der Organisation repräsentiert werden. Das Exekutivbüro umfaßt 7 Mitglieder, die die zwischenzeitlichen Geschäfte führen, wobei dem Präsidenten eine weitgehende Handlungsmacht eingeräumt ist. Durch turnusmäßige Sitzungen in den Städten der Provinz wird versucht, den Problemen einer Zentralisierung in Paris entgegenzuarbeiten, das naturgemäß (so auch im Exekutiv-Büro) ein Ubergewicht hat.

Durch einen besonderen Ausschuß zum Thema "Gruppenpraxis « versucht das Syndikat, die Probleme der berufspraktischen Entwicklung intensiver aufzuarbeiten, und gleichzeitig zu den Problemen der Ausbildung von Anwälten während des Studiums und der Zeit als Stagiaire Stellung zu nehmen.

Das "Syndicat des Avocats de France« erscheint damit als eine Organisation mit gewerkschaftlicher Orientierung, die in den Forderungen zur eigenen Berufspraxis den Stellenwert der Berufsausübung, seine Funktion und Effizienz in der Gesellschaft einbezieht und damit eine Perspektive der Gemeinsamkeit anwaltlicher Interessenvertretung und gewerkschaftlichen Kampfes weist, die auch in diesem

37a Alle im weitesten Sinne als fortschrittlich zu bezeichnenden jurıstischen Organisationen riefen am ${ }_{3} 3$. März 1975 gemeinsam zu eıner Veranstaltung "Vertetdigung der Freiheıten und Polizeımacht« auf. Folgende Organisationen hatten das Flugblatt unterzeıchnet: Assocıatıon Françaıse des Jurıstes Democrates (entspr. Verband demokratischer Juristen); Droit et Démocratıe; Ligue des Droits de l'Homme Mouvement d'Action Judiciaıre; Mouvement des Jurıstes Catholiques; Association des Magıstrats Réststants; Syndicat de la Magıstrature; Fédératıon Natıonale des Unıons de Jeunes Avocats; Syndicat des Avocats de France. 
Sektor unnötige Schranken der Kommunikation zwischen an gesellschaftlicher

Veränderung orientierten Mitgliedern sogenannter freier Berufe und der lohnabhängigen Mehrheit der Bevölkerung abbaut.

b) Neben diesen kollektiven Formen anwaltlicher Interessenvertretung haben sich in Paris und Lyon zwei Anwaltssozietäten entwickelt, die in einer Zusammenarbeit mit Gewerkschaften und Mieterverbänden versuchen, die drei großen Probleme der Anwaltschaft zu lösen: die Ineffizienz überalteter Berufspraxisstrukturen, die politische Legitimation anwaltlicher Arbeit und die finanzielle Sicherstellung der anwaltlichen Arbeitsbedingungen.

Ziel der in Paris zusammenarbeitenden Anwälte war es, für die Mehrheit des Anwaltsstandes und insbesondere für die jungen Anwälte ein soziales Engagement für die Mehrheit der Bevölkerung praktisch in der Weise umzusetzen, daß diese Arbeit sozial-politisch effektiv, den besonderen persönlichen Bedingungen der Arbeiter und Angestellten angepaßt war und trotzdem eine ausreichende Rentabilität aufwies, ohne daß dabei die juristische Distanz zwischen Engagement und Komplizität verloren ging, durch die das anwaltliche Auftreten vor Gericht ein besonderes Gewicht erhält.

Um die anwaltliche Arbeit zu effektivieren, anwaltliche Dienstleistungen für die große Mehrheit der Bevölkerung zu verbessern und politisches und soziales Engagement mit der beruflichen Tätigkeit zu verbinden, wurde in Paris das Modell einer Gruppenpraxis mit dezentralen Büros in den Arbeiterbezirken bei zentraler Verwaltung und arbeitsteiliger Kooperation in enger Zusammenarbeit mit Gewerkschaften und Mieterorganisationen verwirklicht.

Dreizehn Anwälte arbeiten auf der Basis individueller Gleichberechtigung zusammen, wobei entsprechend dem Alter und der Berufserfahrung fixe Grundeinkommen pro Monat vereinbart sind, zu denen je nach der monatlichen Bilanz durch gemeinsamen Beschluß prozentuale Aufschläge hinzukommen können. Die neun Anwälte arbeiten in fünf Anwaltbüros, neun in vier Büros in den Arbeitervororten von Paris und vier in einem Büro, das am Sitz der Metallarbeitergewerkschaft unterhalten wird. Jedes Büro beschäftigt ein oder mehrere Schreibkräfte bzw. Sekretärinnen. Demgegenüber ist die Verwaltung so weit arbeitsteilig verteilt, daß für die Buchhaltung nur zwei Personen, die nebenher auch juristische Vertretung in Sachen machen, in denen kein Anwaltszwang besteht, beschäftigt sind, und nur drei Büros einen Bürovorsteher haben, der auch an der Rechtsberatung teilnimmt.

Obwohl die Anwälte in einer Société civil professionel d'avocats eine Gesellschaft bilden, existieren bisher praktisch zwei Einheiten, die jweils unter der Leitung eines der beiden Gründer weitgehend selbständig sind. Jede Einheit hat ihr eigenes Abrechnungs- und Einkommenssystem und die Zusammenarbeit zwischen den beiden Einheiten erfolgt informell (Austausch von Terminsvertretungen, Ubernahme gewerkschaftlicher Rechtsberatung, Sprechstundenvertretung). Dagegen besteht innerhalb jeder Einheit eine starke verwaltungsmäßige Zusammenfassung. Neben gemeinsamer Buchhaltung, Anschaffung der Ausrüstung werden gegenseitig Terminsvertretungen ausgetauscht, die Rechtsberatung von allen Rechtsanwälten unabhängig vom Beratungsort gleichmäßig wahrgenommen und die Ehescheidungs- und Strafsachen durch Aktenaustausch von spezialisierten Anwälten behandelt.

Eine ganz wesentliche Grundlage dieser Anwaltssozietät ist ihr Verhältnis zu den Gewerkschaften. Hier hatte die langjährige Zusammenarbeit eines Anwalts mit der Gewerkschaft in der Resistance, aber auch die eigene gewerkschaftliche Orientierung der Anwälte geholfen, das notwendige Vertrauensverhältnis zu schaffen.

Die Gewerkschaften vermitteln der Anwaltssozietät ihre Klientel, jedoch nicht durch direkte Verweisung, sondern vermittelt über den gewerkschaftlichen Ent- 
scheidungsprozeß. Die Gewerkschaftsmitglieder wenden sich an die Rechtsschutzsekretäre ihres Bereiches. Diese Sekretäre sind als Funktionäre unmittelbar in der gewerkschaftlichen Arbeit im Betrieb aktiv und sollen dementsprechend versuchen, Konflikte mit den Unternehmern mit gewerkschaftlichen Mitteln auszutragen, sei es über das "comité d'entreprise« oder die unmittelbare Solidarität der Kollegen bei Entlassungen, Rückstufungen, Versetzungen etc. ${ }^{38}$ Erst, wo entweder der Fall so kompliziert ist, daß eine Solidarität schwer vermittelbar oder aber allein aussichtslos erscheint bzw. wo es um nicht verallgemeinerbare Einzelfälle geht, wird die Anwaltsgruppe eingeschaltet. Dies erfolgt dadurch, daß dem Anwaltsbüro bereits ausgefüllte Fragebögen über die wichtigen Daten zum Fall zugeleitet werden und den Betroffenen, soweit dies zur weiteren Aufklärung notwendig ist, ein Termin beim Anwalt gegeben wird. Dieser hält seine Sprechstunden in den verschiedenen Gewerkschaftshäusern ab, soweit es sich nicht um Fälle aus seinem Bezirk handelt. Die weitere Behandlung des Falles erfolgt dann im Verhältnis Anwalt-Klient bei ständigem Kontakt zur Gewerkschaft. Im Gegensatz zum DGB übernehmen die französischen Gewerkschaften nicht die Kosten der Rechtsverfolgung außer in ausgesuchten Fällen, bei denen in Zusammenarbeit mit den Anwälten Musterprozesse und Prozeßkampagnen zur Änderung der Rechtsprechung durchgeführt werden ${ }^{39}$.

Außerdem werden der Sozietät Räume in der Rechtsabteilung der Gewerkschaftszentrale zur Verfügung gestellt. Die Gegenleistung der Sozietät besteht darin, daß die Anwälte in den Gewerkschaftshäusern der Außenbezirke - jeweils nach Stunden bezahlt - Rechtsberatung übernehmen, bei der Laienrichter- und juristischen Funktionärsschulung mitwirken und ihr Honorarsystem, das in Frankreich nicht tarifiert ist, gewerkschaftsfreundlich gestalten. So gilt für Gewerkschaftsmitglieder für die Rechtsvertretung ein einheitlicher Gebührensatz in allen Büros von ca. 100,- DM, der jedoch im Einzelfall den sozialen Verhältnissen angepaßt werden kann. Geht der Prozeß verloren, so bleibt es bei dieser Bezahlung, in der die Gerichtsgebühren enthalten sind. Wird der Prozeß gewonnen, so wird ein Prozentsatz der eingeklagten Summe als Honorar vereinbart, wodurch die ungewissen Segnungen der Justiz zumindest etwas gerechter auf die Summe der rechtsuchenden Gewerkschaftsmitglieder verteilt werden. Im Prinzip des gegenseitigen Nutzens sind Gewerkschaft und Anwaltspraxis bei finanzieller und organisatorischer Eigenständigkeit doch eng verbunden.

Bei den Anwälten in den Vororten ist der Zusammenhang mit den Gewerkschaften schon durch die räumliche Trennung gelockert. Dort können sich die Arbeiter auch direkt in den Sprechstunden melden, ohne die Gewerkschaft vorher zu konsultieren, wenngleich auch hier der andere Weg überwiegt. Die Anwälte arbeiten darüberhinaus mit dem Mieterverband und allen ortsgebundenen Interessenverbänden zusammen. Obwohl dort alle Probleme, mit denen sie durch die Ortsnähe konfrontiert sind, wie Ehescheidung, Unterhalt, Verkehrsrecht und Strafrecht behandelt werden, macht doch das Arbeitsrecht wegen seiner vielfältigen Präsenzpflichten bei Gericht sowie der Unsicherheit der Termine $\%$ des Arbeitsanfalls aus, ohne daß dem ein entsprechender Anteil am Gesamteinkommen gegenübersteht.

38 Dies kann, wie bei Renault 1975 geschehen, bis zum Streik in der gesamten Metallbranche bei der Entlassung von ${ }_{13}$ Kollegen führen, wodurch ohne Einschaltung der Gerıchte deren Wiedereınstellung sowie darüberhınaus erreıcht wurde, daß Renault im Gegensatz zu deutschen Automobilfirmen in der sogenannten Auto-Krise keıne Arbeiter entlassen hat, sondern Gewmnembußen in Kauf nahm.

39 So z. B. zur Bekämpfung der CFT, einer von Berliet, Citroen sowte Chrysler-Simca gegründeten und unterstützten gelben Gewerkschaft, der bereıts in emem Prozeß die Tariffähıkest abgesprochen worden 1st. Chavret a. a. O., (Anm. 1), S. 25. 
Das sektorielle Defizit wird jedoch bewußt in Kauf genommen und durch andere Bereiche (Ehescheidungen) sowie lukrative Großprozesse (z. B. Schadensersatz bei Betriebsunfällen) kompensiert.

Die Anwälte treffen sich in regelmäßigen Abständen nicht nur, um die organisatorischen Fragen zu besprechen, sondern vor allem auch, um die rechtliche Umsetzung ihrer politisch-sozialen Konzeption theoretisch zu diskutieren. Eine weitere Diskussion ergibt sich aus den in größeren Abständen erfolgenden Tagungen der Gewerkschaft mit allen für sie arbeitenden Rechtsanwälten, in denen vor allem mit den allein praktizierenden Anwälten die verschiedenen Vorstellungen durchgesprochen werden. Auf der theoretisch-publizistischen Ebene wird die Arbeit dadurch gefördert, daß ein promovierter Jurist vom Anwaltsbüro bezahlt wird, der neben seiner Beratungstätigkeit juristische Fachaufsätze zu Themen schreibt, die die tägliche Praxis im Arbeitsrecht betreffen. Dabei kommt der gewerkschaftlichen Orientierung zugute, daß die Gewerkschaft selber mit einer angesehenen arbeitsrechtlichen Zeitschrift (Revue pratique du droit social = RPDS) verbunden ist.

Die Anwälte, deren Modell von der Gewerkschaft zunächst nur »wohlwollend beobachtet wird «, befinden sich in einem ständigen Diskussionsprozeß, in dem die Rückkehr zur Einzelpraxis noch lange nicht ausgeschlossen ist. Diejenigen, die für einen weiteren Ausbau plädieren, streben eine vollständige Zentralisation der Verwaltung an, bei der die Zusammenarbeit mit den Gewerkschaften gefestigt und die Stadtrandbüros auf Sprechstundenzimmer reduziert werden sollen. Auf überregionaler Ebene $4^{40}$ wird eine Zusammenarbeit mit ähnlichen Sozietäten angestrebt. Diese neue Form anwaltlicher Berufspraxis hat bei den Gerichten und Kollegen keineswegs zu einem Autoritätsverlust geführt, wenngleich diese Autorität nicht mehr so sehr aus der standespolitischen Achtung, sondern aus der Spezialisierung und dem Zusammenhang mit der Macht der Gewerkschaften herrührt. Gleichwohl stößt diese Arbeitsform auch bei sozial engagierten, vor allem älteren Anwaltskollegen auf starkes Mißtrauen, da dieses Modell scheinbar mit der Idee anwaltlicher Unabhängigkeit kollidiert, während auf der anderen Seite gerade die Entscheidungsautonomie, die sich dieses Büro sowohl inhaltlich als auch finanziell vorbehalten hat, bei den Gewerkschaftsfunktionären Ressentiments hervorruft. Ebenso wie die Richter des SM haben diese Anwälte, die im übrigen im SAF organisiert sind, erkannt, daß es angesichts der tatsächlichen Entwicklung der anwaltlichen Praxis, wie sie oben beschrieben wurde, kein Zurück zum 19. Jahrhundert mehr gibt, daß vielmehr eine solche Nostalgie die Chance verspielt, die neuen gesellschaftlichen Strukturen in materieller Unabhängigkeit zu bewältigen. Demgegenüber kann in diesem Modell durch die gewerkschaftliche Zusammenarbeit auf einen Zuwachs an finanzieller Sicherheit und damit materieller Freiheit verwiesen werden, der den Anwälten die Möglichkeit gibt, der größtmöglichen Freiheit beruflicher Tätigkeit innerhalb der bestehenden Verhältnisse näher zu kommen, nämlich ihre Arbeit nicht mehr allein durch die Bestimmung des Entgelts, sondern durch die eigene Uberzeugung zu determinieren.

Der Vorteil dieser neuen Form besteht vor allem auch darin, daß die neuen Abhängigkeiten, die die herrschenden Formen über das Entgelt verschleierter inhaltlicher Abhängigkeit aufheben, offen liegen und vom Anwalt jeweils eine bewußte und verantwortliche Entscheidung erfordern. Anwaltliche Unabhängigkeit kann sich auf die Dauer nicht durch das Leugnen faktischer Abhängigkeit, sondern

40 Schon heute werden durch Anwälte dieser Sozıetät wichtıge Prozesse der Metall-Gewerkschaft in ganz Frankreıch vertreten, insbesondere auch, wenn die ortsansässıgen Anwälte gegen stadtbeherrschende Unternehmen aus beruflichen Rücksıchten nıcht mıt dem notwendigen Nachdruck vorgehen können. 
nur durch die bewußte Wahl zwischen den verschiedenen Abhängigkeitsverhältnissen herstellen, ohne die anwaltliche Arbeit tatsächlich heute nicht mehr geschieht. Diese Abhängigkeit praktisch zu überwinden, ist nur noch in einem Zusammenschluß von Anwälten möglich, die es verstehen, das Vertrauen der in vielfältigen Formen der Interessenvertretung organisierten Bürger zu erwerben. Erst die Beziehung zu den bestehenden und entstehenden Interessenorganisationen gewährleistet, daß anwaltliche Arbeit in materieller Sicherheit ihren Teil zur Verwirklichung sozialer Gleichheit beiträgt und weder in Komplizität noch in sozialer Bevormundung unbewußter Klienten verharrt. 